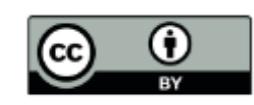

\title{
Integração entre atenção primária à saúde e atenção especializada: paralelos entre a experiencia Brasileira e Portuguesa*
}

\section{Integración de la atención primaria a salud y la atención especializada: paralelos entre la experiencia brasileña y la portuguesa}

\section{Integrating the Primary Health Care and the Specialized Health Care: Parallels between the Brazilian and the Portuguese Experiences}

Recepção: 25 de março de 2019. Aprovação: 03 de outubro de 2019.

Publicação: 30 de junho de 2020.

DOI: https://doi.org/10.11144/Javeriana.rgps19.iaps

\author{
Alexandre Morais Nunes ${ }^{a}$ \\ Universidade de Lisboa, Portugal \\ ORCID: https://orcid.org/https://orcid.org/0000-0002-6808-7769
}

Como citar este artigo: Nunes A. Integração entre atenção primária à saúde e atenção especializada: paralelos entre a experiencia Brasileira e Portuguesa. Revista Gerencia y Políticas de Salud. 2020;19. https://doi.org/1 0.11144/Javeriana.rgps19.iaps

\footnotetext{
a Autor correspondente. Correio eletrônico: anunes@iscsp.ulisboa.pt
} 


\section{Resumo}

O presente artigo tem por objetivo analisar os processos desenvolvidos por Brasil e Portugal para integrar a Atenção Primária à Saúde e a Especializada. Entre as iniciativas mais usuais se destacam a conceção da atenção primários como porta de entrada no sistema sendo atribuído papel de destaque ao médico de família, a territorialização dos serviços por proximidade geográfica saúde, o encaminhamento para consultas de especialidade e a adoção de protocolos clínicos consensuados entre ambas as partes. Como principais resultados verificou-se que, no Brasil, ocorreu a implantação de sistemas descentralizados de regulação, e, em Portugal, a criação de unidades locais de saúde (estruturas do ministério da saúde que integra a atenção primária e especializada com uma mesma equipe de gestão). Em conclusão, destaca-se o desafio da necessidade de desenvolvimento de uma política de verdadeiro investimento para reforçar a capacidade de resposta da atenção especializada e para valorizar mais o trabalho dos profissionais da atenção primária, reforçando a cultura de colaboração que contribuíram para o êxito dos processos de integração com resultados visíveis no estado de saúde e satisfação dos usuários.

Palavras-chave: atenção primária à saúde, assistência hospitalar, integração de sistemas, continuidade da assistência ao paciente, assistência integral à saúde, Brasil, Portugal.

\section{Resumen}

El presente artículo tiene como objetivo analizar los procesos desarrollados en Brasil y Portugal para integrar la Atención Primaria a Salud y la Especializada. Entre las iniciativas más usuales se destaca la concepción de la atención primaria como puerta de entrada al sistema atribuyéndose un papel predominante al médico de familia, a la territorialización de los servicios por proximidad geográfica salud, la remisión a consultas con especialistas y la adopción de protocolos clínicos consensuados entre ambas partes. Como principales resultados se verificó que, en Brasil, se implantaron sistemas descentralizados de regulación y, en Portugal, se crearon unidades locales de salud (estructuras del ministerio de salud que integran la atención primaria y la especializada con un mismo equipo de gestión). En conclusión, se destaca el reto de la necesidad de desarrollo de una política de verdadera inversión para reforzar la capacidad de respuesta de la atención especializada y para valorizar más el trabajo de los profesionales de atención primaria, reforzando la cultura de colaboración que contribuya al éxito de los procesos de integración con resultados visibles en el estado de salud y satisfacción de los usuarios.

Palabras clave: Atención primaria a la salud, asistencia hospitalaria, integración de sistemas, continuidad de la asistencia al paciente, asistencia integral a la salud, Brasil, Portugal.

\section{Abstract}

This paper aims to analyze the processes developed in Brazil and Portugal intended to integrate the Primary Health Care and the Specialized Health Care. Primary Health Care conceived of as the first step for accessing to the health system stands out among the most usual initiatives and thus the family doctors are attributed a prevailing role. It is also emphasized the territorialization of the health services based on the geographic location, the referral to specialized doctors and the implementation of clinic protocols agreed between the parties. The main results indicate that in Brazil they implemented decentralized regulatory systems, and, in Portugal they created local health units (structures owned by the health ministry that integrate primary health care and specialized health care provided by the same management team). In conclusion, this work underlines the need to develop a true investment policy intended to strengthen the response capacity in the specialized health care and to add more value to the work performed by the primary care professionals. This way, they will strengthen the collaborative culture that will translate into successful integration processes with results such as good health and user's satisfaction.

Keywords: primary health care, hospital care, systems integration, continuity in the patient care, integral health care, Brazil, Portugal. 


\section{Introdução}

Os sistemas públicos de saúde em todo o mundo objetivam a garantia do direito de proteção da saúde e são influenciados por contextos humanos, ambientais, culturais, políticos. Po risso, cada vez mais as necessidades em saúde dos cidadãos são imprevisíveis, sendo exigido aos sistemas de saúde a preparação adequada para uma resposta global (em todos os níveis de assistência) para garantir a continuidade da assistêmcia a todos os pacientes (1).

Brasil e Portugal são países desenvolvidos que se caracterizam pela existencia de sistemas nacionais de saúde que apresentam principios identivos desde logo por apresentarem uma cobertura universal, serem financiados por impostos e por terem iniciado reformas da atenção primária (principal porta de entrada no sistema de saúde) desde os anos 80 e 90 sempre para construir uma melhor resposta na assistêmcia aos pacientes, dada a proximidade e o papel fundamental desenvolvido pelo médico de família e sua equipe. Porém, ambos os países apresentam ainda pontos de melhoria nomeadamente em termos do acesso e da qualidade, o que é comum aos demais sistemas públicos universais de saúde um pouco por todo o mundo, mas

que é necessário combater. A Tabela 1 apresenta outros indicadores que caracterizam diferenças entre os sistemas de saúde brasileiro e português. 
Alexandre Morais Nunes

Tabela 1. Características dos sistemas de saúde - Brasil e Portugal

\begin{tabular}{ccc}
\hline & Brasil & Portugal \\
\hline Cobertura & $\begin{array}{c}\text { Pública universal (de direito, não } \\
\text { de fato) }\end{array}$ & $\begin{array}{c}\text { Pública universal (de direito, não } \\
\text { de fato) }\end{array}$ \\
\hline $\begin{array}{c}\text { Fonte principal de financiamento } \\
\text { Gasto em saúde em \% do Produto } \\
\text { Interno Bruto }\end{array}$ & $\begin{array}{c}\text { Público (impostos gerais) } \\
\text { Gúblico (impostos gerais) }\end{array}$ & $9,1(2013)$ \\
\hline $\begin{array}{c}\text { Gasto total em saude per capta } \\
\text { (\$US PPP) }\end{array}$ & $6,2(2013)$ & $2535,9(2013)$ \\
\hline $\begin{array}{c}\text { \% população coberta por seguros } \\
\text { de saúde privados) }\end{array}$ & $994,8(2013)$ & $25 \%(2017)$ \\
\hline $\begin{array}{c}\text { Expectativa média de vida } \\
\text { Taxa bruta de mortalidade } \\
\text { (100.000 habitantes) }\end{array}$ & $74,7(2015)$ & $1022(2015)$ \\
\hline
\end{tabular}

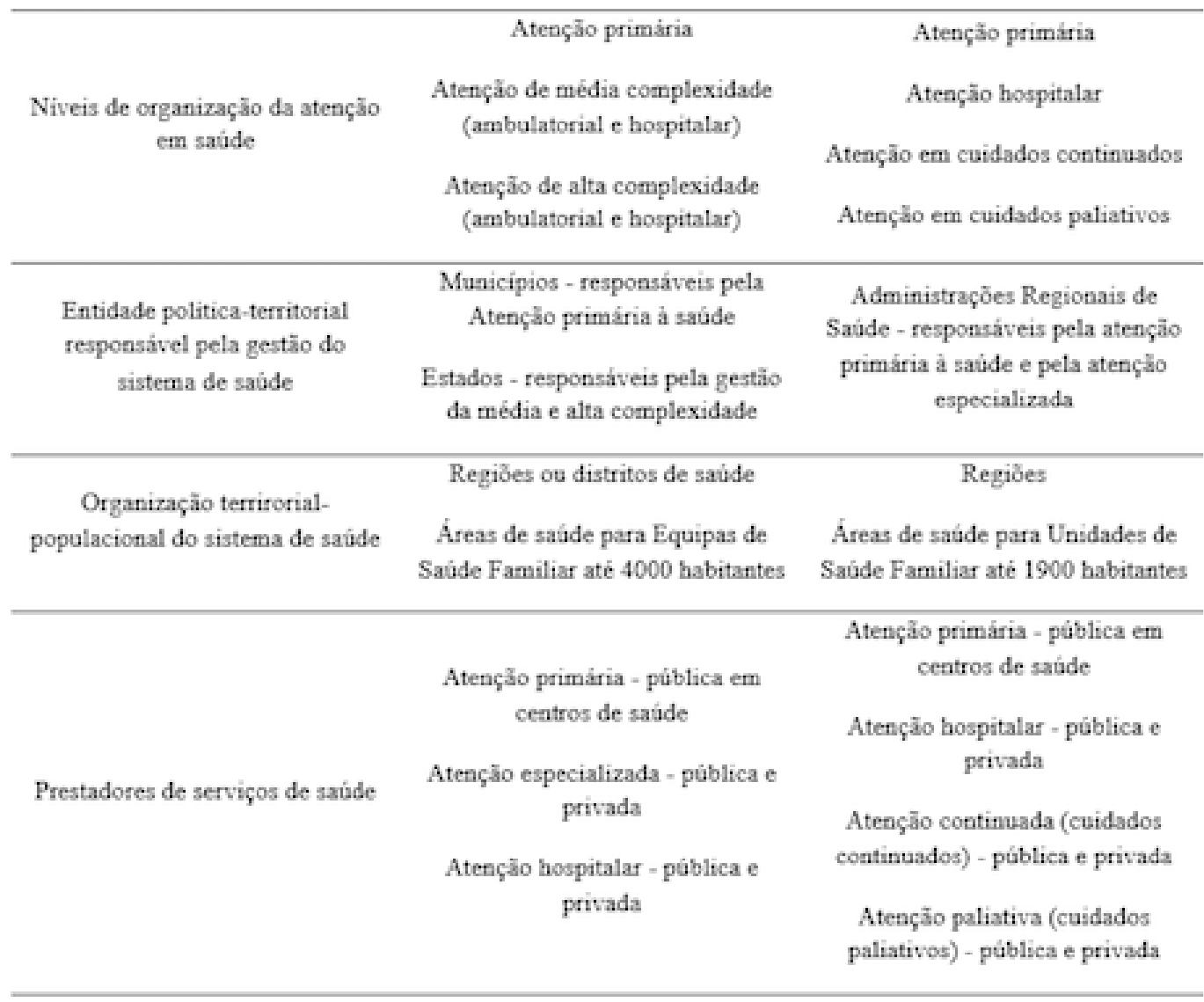

Fuente: Simões et al. (2), OCDE Health Status (3), Almeida et al. (4). 
Tabela 1. Características dos sistemas de saúde - Brasil e Portugal (continua)

\begin{tabular}{|c|c|c|}
\hline & Brasil & Portugal \\
\hline Vinculo laboral & $\begin{array}{c}\text { Diversos regimes: servidores } \\
\text { públicos, regime CLT } \\
\text { (consolidação das leis do trabalho), } \\
\text { contratos temporais }\end{array}$ & $\begin{array}{l}\text { Diversos regimes: servidores } \\
\text { públicos, regime CIT (contrato } \\
\text { individual de trabatho), contratos } \\
\text { temporais; prestações de serviços } \\
\text { (contratos de trabalhador } \\
\text { indepeadente) }\end{array}$ \\
\hline Médicos por 10.000 habitantes & $18,2(2010)$ & $35,2(2010)$ \\
\hline $\begin{array}{l}\text { Enfermeiros por } 10.000 \\
\text { habitantes }\end{array}$ & $14,7(2010)$ & $56,8(2010)$ \\
\hline $\begin{array}{c}\text { Leitos hospitalares por } 10.000 \\
\text { habitantes }\end{array}$ & $22,9(2012)$ & $34,1(2012)$ \\
\hline $\begin{array}{l}\text { Número médio de consultas } \\
\text { médicas anuais por habitante }\end{array}$ & $2,8(2012)$ & $4,1(2012)$ \\
\hline
\end{tabular}

Fuente: Simões et al. (2), OCDE Health Status (3), Almeida et al. (4).

O Sistema Único de Saúde (SUS) implantado no Brasil foi um ponto fundamental para a promoção da resposta em saúde e ao desenvolvimento do modelo assistencial onde atualmente se encontra o Programa Saúde da Família, inicialmente visto como um programa de saúde vertical, foi posteriormente considerado pelo Governo Brasileiro como a principal estratégia de reforma da atenção à saúde. Esse programa, desde seu inicio, sempre objetivou a promoção do acesso universal à atenção primária por meio de uma aposta na Estratégia de Saúde da Família (ESF) que além da realização de consultas integrou atividades de promoção, educação em saúde, prevenção de agravos, intervenção social sendo considerada uma porta de entrada no sistema e constituída por uma equipe multiprofissional altamente treinada e diferenciada (5).

O Serviço Nacional de Saúde português foi criado em 1979, e representou um grande passo na atenção à saúde, por apresentar uma cobertura universal (para todos os cidadãos), geral (em todas as necessidades) e gratuito (financiado por impostos e sem qualquer custo para os cidadãos) (6). Todas as características do Serviço Nacional de Saúde sobreviveram até aos dias de hoje (quase 40 anos) com exceção da gratuidade que passou desde 1989 a ser tendencial pela introdução de copagamentos (taxas moderadoras) para regular e moderar o acesso que por vezes era abusivo por parte de muitos cidadãos.

A atenção primária em Portugal inclui unidades integradas no SNS presta assistência na promoção da saúde, na prevenção da doença, e na gestão nas situações de saúde agudas, por meio de uma abordagem centrada na pessoa, familia e comunidade. Como exemplos de atividades se destacam: cuidados médicos gerais para a população adulta; cuidados pré-natais; cuidados 
infantis; saúde das mulheres; planejamento familiar e cuidados perinatais; primeiros socorros; certificação da incapacidade de trabalhar; visitas domiciliares; serviços preventivos, incluindo imunização e triagem para câncer de mama e cervical e outras doenças evitáveis. A gestão e o financiamento da atenção primária é realizada pelo Estado através de Agrupamentos de Centros de Saúde (7).

A importância da atenção primária à saúde foi retratada e identificada em 1978 por vários países através da Declaração de Alma-Ata e foi reforçada em 2018 novamente no Cazaquistão onde foi assinado um novo comprimisso, a Declaração de Astana. Este documento reforçou a necessidade de integrar a saúde em todos os setores de atividade, de construir rede de atenção primária sustentável e proxima da população, de capacitar os cidadãos e as comunidades e ainda alinhas e de integrar a atenção primária nas políticas, estretégias e planos nacionais de saúde.

No entanto, existem ainda alguns problemas assistenciais na atenção primária e que condiciona a atenção hospitalar pelo uso excessivo do departamento de emergência, nomeadamente: a distribuição desigual dos recursos; acesso limitado aos serviços de saúde para pessoas mais pobres e geograficamente isoladas; e falta de profissionais de saúde como por exemplo médicos especializados em Medicina Geral e Familiar (2) Para melhorar esses indicadores desde 2006 foi iniciada a criação de Unidades de Saúde Familiar que na mesma lógica das ESF pretendem aproximar à assistência aos cidadãos não apenas na vertente curativa mas também numa lógica de continuidade da assistência ao paciente com promoção da saúde e prevenção da doença.

A atenção secundária (ou diferenciada) é a mais especializada no SNS português e é prestada em hospitais, centros hospitalares (grupo constituído horizontalmente por unidades hospitalares próximas), e unidades locais de saúde (fusão entre hospitais e unidades de atenção primária). Os hospitais públicos portugueses prestam assistência ao nível da internação, emergência, cirurgia, consulta, tratamentos em hospital de dia, e ainda dispõem de um centro de diagnóstico onde são realizados exames de imagiologia/radiologia e análises clínicas (8).

Um grande passo dado para a integração da assistência da atenção primária à saúde com a atenção especializada foi conseguida por meio de um modelo de gestão inovador, a Unidade Local de Saúde. Este modelo tinha por objetivo proporcionar aos usuários de um determinado território uma assistência integrada e continuada com elevada qualidade e com facilidade de encaminhamento, comunicação e acesso a consultas e exames (9). Neste modelo, a equipe de administração detém as competências de gestão estratégica em ambos os níveis de assistência (10).

Quanto aos cuidados pós-hospitalares, ao longo dos anos o Serviço Nacional de Saúde foi evoluindo e cobria cada vez mais necessidades de saúde. Além da atenção primária e especializada, desde 2006, o sistema passou também a cobrir necessidades de saúde de 
reabilitação e ainda cuidados paliativos que cada vez mais são necessários (5). A tabela seguinte apresenta uma comparação do processo de reforma da atenção primária no Brasil e em Portugal.

Tabela 2. Características da Atenção Primária à Saúde - Brasil e Portugal

\begin{tabular}{|c|c|c|}
\hline & Brasil & Portugal \\
\hline $\begin{array}{c}\text { Processo de reforma da Atenção } \\
\text { Primária à Saúde }\end{array}$ & $\begin{array}{l}\text { Iniciada em } 1994 \text { com a criação } \\
\text { do Programa de Saude Familiar }\end{array}$ & $\begin{array}{l}\text { Iniciado em } 1983 \text { com a criação dos } \\
\text { centros de saúde de segunda geração } \\
\text { Reforçada em } 2005 \text { com a criação das } \\
\text { Unidades de Saúde Familiar }\end{array}$ \\
\hline $\begin{array}{l}\text { Atenção primária como porta de } \\
\text { entrada (filtro) }\end{array}$ & $\begin{array}{c}\text { Proposta em } 1994 \text { em áreas } \\
\text { cobertas com Estratégia Saíde } \\
\text { da Familia }\end{array}$ & $\begin{array}{c}\text { Proposta desde anos } 70 \text { mas nunca } \\
\text { realizada. Os pacientes vão direto no } \\
\text { hospital } \\
\text { Proposto e com resultados positivos } \\
\text { em áreas cobertas com Unidades de } \\
\text { Saúde Familiar }\end{array}$ \\
\hline Co-pagamento por consultas & Não & Sim (taxas moderadoras desde 1992) \\
\hline $\begin{array}{c}\text { Territórios geograficamente } \\
\text { definidos }\end{array}$ & $\begin{array}{c}\text { Somenta para áreas cobertas pela } \\
\text { ESF }\end{array}$ & Sim para todo o pais \\
\hline Composição das Equipes & $\begin{array}{l}\text { Médico generalista } \\
\text { Enfermeiro } \\
\text { Auxiliar de enfermagem } \\
\text { Agentes comunitários } \\
\text { Equipe de saude bucal }\end{array}$ & $\begin{array}{c}\text { Médico especialista em Medicina de } \\
\text { Familia e comunidade (Medicina } \\
\text { Geral e Familiar em Portugal) } \\
\text { Enfermeiro } \\
\text { Auxiliares de ação médica } \\
\text { Psicólogo } \\
\text { Nutricionista } \\
\text { Equipe de saúde bucal (médico e } \\
\text { higienista) } \\
\text { Assistente social }\end{array}$ \\
\hline $\begin{array}{l}\text { Lista de população por médico } \\
\text { de familia }\end{array}$ & $\begin{array}{l}\text { Não (população adscrita à } \\
\text { Equipe e não ao médico ( } 3000 \text { - } \\
40000 \text { hab) por equipa }\end{array}$ & $\begin{array}{l}\text { Sim. Cada equipa tem médico e está } \\
\text { limitada a um máximo de } 1900 \\
\text { pacientes por lista }\end{array}$ \\
\hline Livre escolha do médico & Não & $\begin{array}{l}\text { Não (o médico é atribuido) a troca de } \\
\text { médico tem de ter nota justificativa e } \\
\text { tem de ser aceite }\end{array}$ \\
\hline Sistema de pagamento & Salário & Salário \\
\hline $\begin{array}{l}\text { Horário normal do } \\
\text { funcionamento dos centros de } \\
\text { saúde }\end{array}$ & $\begin{array}{c}8: 00-17: 00 \text { (segunda a sexta- } \\
\text { feira) }\end{array}$ & $\begin{array}{l}\text { 8:00-20:00 (segunda a sexta-feira) e } \\
\text { em alguns casos (época de gripe e } \\
\text { verão) nos sabados e algumas } \\
\text { unidades até às } 00 \mathrm{~h}\end{array}$ \\
\hline Carga horária & 40 horas semanais & $\begin{array}{l}42 \text { horas semanais (médico) } \\
35 \text { horas semanais (restante equipa) }\end{array}$ \\
\hline $\begin{array}{c}\text { Formação especializada em } \\
\text { Medicina de Familia e } \\
\text { Comunidade }\end{array}$ & Não obrigatória & Atualmente obrigatória \\
\hline
\end{tabular}

Fuente: Simões et al. (2), Almeida et al. (4).

Passados vários anos desde o inicio da reforma da saúde em Portugal e no Brasil cada vez mais a integração entre a atenção primária e especializada é um desafio. Segundo Santana e Costa (1), a integração em Portugal mesmo com os passos dados no modelo de Unidade Local de Saúde segue ainda como um tema pendente que precisa de melhorias. No Brasil, mesmo com a grande expansão das Equipes de Saúde Familiar ainda não dispõem de um modelo de coordenação que 
permite maximizar todas as suas potencialidades, e no lado da atenção especializada um dos problemas mais referidos na literatura é a baixa oferta que que afeta negativamente o acesso dos pacientes (11).

Segundo a Organização Pan-Americana da Saúde (12), a integração entre a atenção primária e especializada é um solução para a assistência integral à saúde que permite um maior seguimento dos pacientes evitando o agravamento da doença crônica.

Estudos nacionais e internacionais identificam as vantagens da integração um conjunto de reformas realizadas que envolveram processos de integração. Como exemplo, para Sobczak (13) realizado na Polónia concluiu que a integração representa vantagem competitiva essencial para promover uma boa reforma nos sistemas de saúde; Byrne e Ashton (14) que no seu estudo realizado nos Estados Unidos confirmou que a integração vertical pode ser apropriada e gera oportunidades para ganhos de eficiência devido à melhor organização; superarão os custos adicionais e as perdas de eficiência; Leichsenring (15) em um estudo que comparou processos de integração em 9 países europeus (Austria, Dinamarca, Finlandia, França, Alemanha, Grécia, Itália, Holanda e Reino Unido) e conclui que existiram ganhos de qualidade pelo melhor acompanhamento de cada paciente após integração; Tsutsui (16), ao estudar os processos de integração no Japão concluiu que se gerou um maior monitoramento e vigilancia da saúde sobretudo da população mais idosa; Ahgren e Axelsson (17), estudando a experiencia Sueca concluem que o carater agregador da integração supera a fragmentação na assistência à saúde.

No Brasil, Almeida et al. (11) ao estudar a Implementação da Estratégia Saúde da Família em Grandes Centros Urbanos analisaram experiencias consolidades de implantação de ESF, nomeadamente relativas à integração entre níveis assistenciais em Aracaju (Sergipe), Belo Horizonte (Minas Gerais), Florianópolis (Santa Catarina) e Vitória (Espírito Santo), concluiram que a a falta de integração entre diferentes prestadores, foram entraves à garantia do cuidado integral, tornando incompleto o processo de integração da rede. Em 2013, foi realizado por Almeida et al. (4) um estudo comparativo relativo aos processos de integração no Brasil vs Espanha no qual se destacou o carater integrativo como fundamental para o estabelecimento de protocolos de colaboração, com destaque no papel do médico da atenção primária à saúde como mediador dos processos.

Curiosamente não são encontrados estudos sobre a integração realizada em Portugal, pelo que este estudo ganha uma maior relevância acrescida da comparação com o Brasil.

O presente artigo por meio da análise dos processos identifica os instrumentos de integração entre a atenção primária e especializada, comparando a realidade brasileira baseada nos resultados obtidos no estudo de Almeida et al. (4) comparando-os com a realidade portuguesa, permitindo assim uma comparação entre os países e ainda identificar praticas exitosas que podem ser aplicadas/partilhadas dentro das especificidades de cada um dos país. 


\section{Método}

Com o objetivo de analisar as estratégias de integração entre a atenção primária à saúde e a atenção especializada entre Portugal e Brasil, foram comparados os resultados qualitativos coletados em quatro municípios brasileiros no estudo de Almeida et al. (4) com os dados coletados em três regiões de Portugal (Norte, Centro e Alentejo) que até ao momento apostaram no modelo inovador de integração.

Tal como no estudo de Almeida et al. (4) e para ser possível a comparação, aplicou-se o mesmo método de pesquisa em Portugal e buscou-se analisar características, semelhanças e diferenças com relação às estratégias de integração entre atenção primária e especializada, na perspectiva dos gestores da saúde. Para a coleta de informação foram realizadas 45 entrevistas semiestruturadas a gestores e profissionais entre os meses de janeiro e junho de 2018, que foram gravadas e transcritas. Foram selecionados os principais intervenientes nos processos de organização da saúde ao nível da gestão e da prestação em cada região, cobrindo assim todas as dimensões objeto de estudo.

De forma a comparar os dados coletados em Portugal, a apresentação dos resultados foi realizada da mesma forma que no estudo de Almeida et al. (4) realizaram no Brasil, pelo que foi estruturada em quatro dimensões: a) Posição ocupada pela atenção primária à saúde e prioridade da integração entre níveis de atenção nas políticas regionais de saúde; b) Instrumentos de integração nos serviços de saúde; c) Organização dos fluxos para a atenção especializada; d) Cultura de colaboração entre a atenção primária à saúde e a atenção especializada.

Para a análise das informações coletadas nas entrevistas foi realizada uma análise de conteúdo e os resultados foram organizados em categorias previamente definidas e colocadas as seguintes questões:

No seu entendimento, a atenção primária à saúde em Portugal é considerada pela população como o principal meio de acesso. Existe uma outra forma de acesso privilegiada pela população?

A integração de cuidados foi uma política positiva para o acesso à assistência à saúde? De que forma?

Nos locais onde não existe integração, há formas alternativas para melhorar a resposta e a articulação entre atenção primária e especializada?

Relativamente ao encaminhamento dos pacientes. Existe partilha de informação e encaminhamento para realização de exames facilitada nas regiões com integração da assistência?

Nos casos de maior distância, existem soluções eletrónicas que facilitem a comunicação entre atenção primária e especializada? 


\section{Resultados}

Neste ponto apresentam-se os resultados obtidos no estudo de campo realizado em Portugal. Os resultados obtidos no Brasil serão adaptados do estudo realizado por Almeida et al. (4) e serão confrontados com os achados encontrados em Portugal no ponto de discussão. Na pesquisa realizada em Portugal não gerou categorias emergentes, o que se justifica pelas elevadas semelhanças entre os sistemas de saúde no campo da integração.

\section{a) Posição ocupada pela atenção primária à saúde}

$\mathrm{Na}$ opinião de todos os entrevistados, a atenção primária tem um papel central no Serviço Nacional de Saúde sendo considerada a sua principal porta de entrada. Contudo, o serviço de emergência hospitalar é ainda umas das portas mais procuradas e usada muitas vezes por pacientes não urgentes.

\section{b) Prioridade do tema da integração entre níveis de atenção nas políticas locais de saúde}

A integração entre a atenção primária e especializada foi um desafio e prioridade para a reforma da saúde em Portugal, materializada na constituição de Unidades Locais de Saúde. Segundo todos os entrevistados, embora a população coberta seja a mesma, os pacientes têm melhor acesso e mais rápido com a integração pois podem ter mais consultas e um melhor acompanhamento:

[...] a integração na nossa área permitiu uma maior organização das consultas e um maior acesso aos pacientes que se sentem mais acompanhados e são mesmo contactados quando não marcam ou falham um agendamento (Gerente centro de saúde região Centro).

O grande número de pessoas idosas e de pacientes com doença oncológica e outras patologias crônicas gerou uma pressão nas unidades locais de saúde que deram uma melhor resposta e teve uma repercussão nas restantes unidades da atenção primária que passaram a dar máxima prioridade na relação com a atenção especializada: “[...] toda a região apresentou uma melhoria na relação entre a atenção primária e a especializada pois as unidades que ficaram de fora da área do modelo de unidade local de saúde adotaram algumas das novas medidas" (gerente região Norte).

Na base da decisão pela integração da atenção primária e especializada para $75 \%$ dos inquiridos estão os problemas relacionados com os custos dos exames realizados no sector privado e pagos integralmente pelo Estado. Os pacientes da atenção primária não realizavam, exames no hospital que tinha capacidade de resposta mas sem pacientes encaminhados:

[...] a sustentabilidade do Serviço Nacional de Saúde estava em risco pois a gestão dos pedidos de exame não era regulada e os médicos prescreviam duas e três vezes o mesmo exame e o paciente ia sempre ao setor 
privado realiza-los, sendo a fatura passada à Administração Regional de Saúde que tutelava todos os centros de saúde (gestor responsavel pela regulação, controle e avaliação da ARS Centro).

Contudo, $25 \%$ dos entrevistados referem que a principal razão que motivou a integração foi o encaminhamento de pacientes para a atenção especializada e que não tinham consulta pois existiam longas listas de espera (médico da atenção primária). A responsabilidade pela implementação de medidas e estratégias de integração entre a atenção primária à saúde e a atenção especializada é, segundo todos os entrevistados, partilhada por gestores de ambos os níveis de atenção que consultam os médicos e enfermeiros com funções de gestão:

[...] a integração em Portugal funciona pois ambos os níveis são respeitados mutuamente e nenhum se sobrepõe. A principal ideia é estabelecer o ciclo do paciente desde o momento em que entra no sistema (atenção primária) até ao fim do seu percurso (diretor da atenção primária).

Embora exista uma preocupação política local para promover a integração não apenas nas unidades locais de saúde, mas também nas outras unidades e hospitais, ainda existem problemas de acesso. Segundo os gestores (atenção primária, hospital, gestores regionais) e todos os profissionais de saúde entrevistados a resposta ao domicílio ainda não é total e esses pacientes (sobretudo idosos) são prejudicados por não terem sempre o acesso, o que faz com que esses pacientes sejam transportados à emergência hospitalar. Outro caso apontado é o dos pacientes que necessitam de atenção paliativa pois nem sempre as equipas da atenção primária e especializada conseguem dar a resposta adequada e necessária a cada vez mais necessidades "[...] não temos equipas suficientes para fazer visita ao domicilio" (médico da atenção primária); "por vezes chegam à urgência do hospital pacientes que poderiam ser acompanhados em casa" (enfermeiro hospitalar).

Em Portugal, o médico da atenção primária avalia, pede exames de diagnóstico necessários (podem ser realizados no hospital ou em entidade privada) e encaminha para a atenção especializada o que no caso das consultas e cirurgias tem enormes listas de espera. É de destacar que na atenção primária não existem listas de espera, os pacientes urgentes são atendidos por um médico no próprio dia (pode ser o seu médico ou outro) existindo para isso uma modalidade conhecida como "consulta aberta" onde os pacientes recorrem em caso agravamento do seu estado de saúde ou em caso de urgência não emergente. Na região Centro as Unidades Locais de Saúde têm por norma o hábito de encaminhar os pacientes da atenção primária para fazer seus exames no hospital poupando medida essa eficiente que permitiu poupar recursos públicos. Além da poupança, esta medida permite detetar logo casos de pacientes que necessitem de assistência especializada que são logo contactados e, em simultâneo, o médico de família é alertado.

\section{c) Instrumentos de integração dos serviços de saúde}

Desde a criação 2002 com influência do modelo europeu da New Public Management, foram desenvolvidas aplicações informáticas nas unidades de saúde, objetivando sempre a criação de um processo único que permite a partilha de informação entre os vários níveis de atenção. Assim 
existe uma história clínica compartilhada que contém um repositório de informações do paciente acessível a todos os profissionais.

Os entrevistados destacam o desenvolvido um registo eletrónico de saúde que permite ao paciente ter acesso ao seu próprio processo em sua casa por meio de uma ligação segura à internet (password de acesso funciona por serviço de pré-registo numa chave móvel digital). Ou seja a senha é gerada automaticamente para o telemóvel do paciente que a deve digitar no campo próprio da sua página pessoal do Portal do Serviço Nacional de Saúde "área do cidadão". Essa área além da consulta do processo clínico permite ao cidadão saber quem acessou ao seu processo e ainda permite marcar consultas, consultar exames realizados e verificar a medicamentação prescrita.

A troca de informações clínicas ainda que exista um registo único, é obrigatória. A própria aplicação gera um relatório sempre que o paciente é encaminhado da atenção primária para a especializada ou ao contrário da especializada para acompanhamento contínuo na atenção primária. Esse relatório é regular e obrigatório, contém dados sobre o estado de saúde, intercorrências, alertas, medicação prescrita e um campo aberto para os médicos deixarem mensagem de alerta.

\section{d) Organização dos fluxos (da atenção primária para atenção especializada e da especializada para a primária)}

Em Portugal não podemos falar de um único fluxo. Ainda que na integração e sabendo de antemão que a atenção primária é a principal porta de acesso de dê prevalência ao fluxo da atenção primária para a especializada, a verdade é que num modelo de integração o trajeto contrário é igualmente importante. É um exemplo os casos dos pacientes que recorrem aos serviços de urgência hospitalar para casos não emergentes sem qualquer necessidade que deverão ser evitados no futuro ou outros casos como é o exemplo do agravamento da patologia crônica por falta de acompanhamento da atenção primária.

Restringindo como no estudo original de Almeida et al. (4) as questões à organização dos fluxos da atenção primária para a especializada. $O$ processo é realizado por meio de um formulário preenchido pelo médico que resume a história do paciente e inclui a avaliação da necessidade, inclui problema de saúde, sinais, sintomas, exames complementares já realizados e medicações. A solicitação é encaminhada pela aplicação informática que gera um processo com um número de pedido que é recebido por um funcionário administrativo do hospital que registra no sistema que atribui o paciente na agenda de um médico especialista. Em casos urgentes o paciente é de imediato transportado ao hospital entrando pelo serviço de emergência levando essa informação em carta de referência (podendo ser a mesma consultada no computador quando o médico de urgência aceder ao processo). 
Esse processo de referencia, tendo em conta as longas listas de espera que infelizmente se registram em algumas consultas de especialidades, têm circuitos preferenciais que consideram prioritários os casos confirmados ou suspeita de câncer, colocando esses pacientes com elevada prioridade, sendo atendidos por norma em menos de uma semana.

Mais recentemente, desde 2011, os exames de diagnósticos pedidos na atenção primária passaram a ser preferencialmente realizados nos hospitais de referência, o que gerou em alguns casos problemas por demora associada a uma falta de capacidade de resposta: "Se verificaram casos de demora nos resultados dos exames (análises e em exames de radiologia) devido a longas listas de espera e falta de preparação dos hospitais para receberem os pacientes que referenciamos aqui do centro de saúde" (médico da atenção primária).

Os casos que ocorram fora do horário normal de funcionamento do centro de saúde podem recorrer aos Serviços de Urgência Básica (existentes em áreas que distem mais de $50 \mathrm{~km}$ do hospital) que além das normas nacionais para orientação clínica da Direção Geral da Saúde, dispõem que um conjunto de procedimentos realizados em parceria com a atenção especializada para a referenciação dos pacientes para o hospital ou para a consulta, isto ocorre com sucesso nas Unidades Locais de Saúde, onde muitas vezes os médicos da atenção primária integram (fora do horário normal de trabalho) as equipes de emergência e lidam com ambas as realidades.

As listas de espera são monitoradas pela atenção especializada, que tem funcionários que recebem os pedidos provenientes da atenção primária e distribuem pela agenda dos médicos especialistas. É sempre tida em conta, como já referido, a prioridade atribuída que pode ser: normal (até 150 dias), prioritário (até 60 dias) ou muito prioritário (até 30 dias). A informação sobre os tempos médios de espera para atendimento em serviço de urgência, consulta ou cirurgia é publica e disponível para consulta (18).

\section{d) Cultura de colaboração entre Atenção Primária à Saúde e Atenção Especializada}

Ao avaliar o conhecimento dos profissionais sobre os processos realizados em cada um dos níveis de atenção verificou-se que todos conhecem a sua própria realidade e a complementar, ou seja os profissionais que atuam na atenção primária conhecem o processo de trabalho da atenção especializada e estes por sua vez também estar a par dos processos realizados na atenção primária. No entanto, $25 \%$ dos entrevistados refere que os médicos da atenção primária conhecem melhor a atenção especializada pelo fato de lá prestarem assistência em turno de emergência, dando assim a entender que o conhecimento físico é um fator importante para melhorar o processo de integração "[...] nas reuniões de equipe nós gestores e os diretores médicos partilham a sua forma de organização e estamos todos articulados" (gerente Centro); "Eu como médico da atenção especializada nunca tive contacto direto com os colegas da atenção primária no dia a dia, apenas ouço o que o meu diretor me diz" (médico região Norte).

Casos de proximidade não física e de partilha de conhecimento ocorrem por via da telemedicina por meio da qual médicos da atenção primária e especializada podem pedir uma segunda opinião 
e estabelecer uma maior colaboração entre os níveis de assistência "Contatamos os médicos da atenção especializada quando temos um problema ou em casos mais complexos, não sabemos bem para onde encaminhar o paciente" (médico atenção primária Alentejo).

Além dos casos apresentados existem ainda reuniões de equipe e existe partilha de enfermeiros e técnicos que conhecem tanto a atenção primária como a especializada. Em Portugal o médico de família deixou de ser apenas um prescritor e o médico que se limitava a fazer trabalho burocrático passando a ser um especialista, o que valorizou a percepção e o respeito dos pacientes. Este fato ocorre com maior notoriedade nas Unidades Locais de Saúde que por serem mais recentes passaram a admitir médicos mais jovens especializados em Medicina Geral e Familiar (Medicina da Família).

Em resumo, a tabela seguinte apresenta os resultados comparativos entre Brasil e Portugal relativamente às questões colocadas. 
Tabela 3. Resultados do estudo

\begin{tabular}{|c|c|c|}
\hline \multicolumn{3}{|c|}{$\begin{array}{l}\text { Posaça ocupada pela atenç̃o primaria e prioridade do tema da } \\
\text { políticas locais de saúde }\end{array}$} \\
\hline & Brasil* & Portugal \\
\hline $\begin{array}{l}\text { Presenca do tema no debate } \\
\text { da politica de saúde }\end{array}$ & Sim & Sim \\
\hline Importância atribuida (grau) & Elevado & Elevado \\
\hline $\begin{array}{l}\text { Capacidade de intervenção } \\
\text { dos gestores da atenção } \\
\text { primária na implementação } \\
\text { de medidas pró- } \\
\text { cootdenação }\end{array}$ & $\begin{array}{l}\text { Alta - a coordenaçāo é centrada na } \\
\text { Secretaria Municipal de Saúde }\end{array}$ & $\begin{array}{l}\text { Partilhada de igual modo com os } \\
\text { gestores da atençāo especializada } \\
\text { procurando consensos nas medidas }\end{array}$ \\
\hline
\end{tabular}

\begin{tabular}{|c|c|c|}
\hline $\begin{array}{l}\text { Grupos mais afetados por } \\
\text { inadequada coordenação }\end{array}$ & $\begin{array}{l}\text { Crianças, idosos, pacientes crônicos } \\
\text { e pacientes com patologias graves } \\
\text { com dificuldade para acessar os } \\
\text { serviços de saúde }\end{array}$ & $\begin{array}{l}\text { Pacientes idosos com dificuldades de } \\
\text { deslocação e pacientes com } \\
\text { necersidades de cuidados paliativos }\end{array}$ \\
\hline $\begin{array}{l}\text { Porta de entrada no sistema } \\
\text { de saude }\end{array}$ & Unidade de Saúde da Familia & $\begin{array}{c}\text { Todos os centros de sauide da atençäo } \\
\text { primáris } \\
\text { Urgência hospitalar }\end{array}$ \\
\hline $\begin{array}{l}\text { Papel de filtro para a } \\
\text { atenç̄o especializada }\end{array}$ & $\begin{array}{l}\text { Médicos da Equipe de Saúde da } \\
\text { Familia }\end{array}$ & Médico de familia \\
\hline \multicolumn{3}{|c|}{ Instrumentos de integraç̃o dos serviços de saude } \\
\hline & Brasil* & Portugal \\
\hline $\begin{array}{l}\text { História clinica } \\
\text { informatizada }\end{array}$ & Incipiente & $\begin{array}{l}\text { Sim em todas as unidades de atenção } \\
\text { primáris }\end{array}$ \\
\hline História clínica única & $\begin{array}{l}\text { Incipieste (entre atenção primária e } \\
\text { especializada) }\end{array}$ & $\begin{array}{l}\text { Sim, partilbada entre atenção } \\
\text { primária e especializada }\end{array}$ \\
\hline $\begin{array}{l}\text { Retorno das informaçòes } \\
\text { clinicas por meio de } \\
\text { instrumento de } \\
\text { contrareferència }\end{array}$ & $\begin{array}{l}\text { Irregular, com graus distintos de } \\
\text { qualidade }\end{array}$ & $\begin{array}{l}\text { Sim e obrigatória. A própria } \\
\text { aplicação informática gera relatório } \\
\text { que é partilhado entre equipes de } \\
\text { saúde }\end{array}$ \\
\hline
\end{tabular}


Tabela 3. Resultados do estudo (continua)

\begin{tabular}{|c|c|c|}
\hline \multicolumn{3}{|c|}{ Organizaçào dos fluxos } \\
\hline & Brasil* & Portugal \\
\hline $\begin{array}{l}\text { Percurso mais comum do } \\
\text { paciente para acesso à } \\
\text { atenção especializada }\end{array}$ & $\begin{array}{l}\text { Encaminhamento do médico da } \\
\text { equipe de saúde familiar }\end{array}$ & $\begin{array}{l}\text { Encaminhamento do médico de } \\
\qquad \text { familia } \\
\text { Emergência hospitalar }\end{array}$ \\
\hline $\begin{array}{l}\text { Circuitos preferenciais para } \\
\text { referência }\end{array}$ & $\begin{array}{c}\text { Priorização do risco indicado pelo } \\
\text { médico da atençäo primária e } \\
\text { avaliada por equipes de reguladores } \\
\text { da SMS }\end{array}$ & $\begin{array}{l}\text { Em casos de suspeita de doença } \\
\text { oncológica segundo critério de } \\
\text { avaliação do médico de famila }\end{array}$ \\
\hline $\begin{array}{c}\text { Estruturas de Atenção } \\
\text { Primária com atendimento à } \\
\text { urgêsocia }\end{array}$ & Naึo & $\begin{array}{l}\text { Sim, apenas existem em regiões mais } \\
\text { remotas (mais de } 50 \mathrm{~km} \text { do bospital) } \\
\text { conhecidas como "Serviços de } \\
\text { Atendimento Básico" } \\
\text { Ėm casos emergentes o médico de } \\
\text { familia chama as equipes de atenç̃o } \\
\text { pré-hospitalar que dispöem de } \\
\text { viaturas com meios e equipamentos } \\
\text { de emergência que asseguram a } \\
\text { deslocação e acompanhamento para o } \\
\text { hospital }\end{array}$ \\
\hline Guias de prática clinica & $\begin{array}{l}\text { Sim, por patologia e ciclos de vida e } \\
\text { protocolos para definição de fluxos e } \\
\text { regulação para atenção especializada }\end{array}$ & $\begin{array}{l}\text { Não especificos. Existem normas de } \\
\text { orientação clinica realizadas pela } \\
\text { Direcĩo Geral da Saude aplicáveis } \\
\text { em todos os niveis. } \\
\text { De forma isolada as UI.S } \\
\text { estabelecem esses protocolos por } \\
\text { patologia para facilitara referenciação }\end{array}$ \\
\hline $\begin{array}{l}\text { Monitoramento das } \\
\text { referèncias realizadas pelos } \\
\text { profissionais da atençīo } \\
\text { primária para a } \\
\text { especializada }\end{array}$ & Nảo & $\begin{array}{l}\text { Sim, os profissionais acompanham e } \\
\text { gerem as listas de espera entando } \\
\text { atentos a desistências e a óbitos dos } \\
\text { pacientes em espera dando lugar a } \\
\text { outros (mantendo a ordem de } \\
\text { prionidade) }\end{array}$ \\
\hline
\end{tabular}


Tabela 3. Resultados do estudo (continua)

\begin{tabular}{|c|c|c|}
\hline \multicolumn{3}{|c|}{ Cultura de colaboração entre atençảo primária e especializada } \\
\hline & Brasil* & Pertugal \\
\hline $\begin{array}{l}\text { Conhecimento do trabalho } \\
\text { desenvolvido em atençio } \\
\text { primíria pelos profissionais } \\
\text { de atençáo especializada }\end{array}$ & Baixo & $\begin{array}{c}\text { Alto. Os profissionais conbecem o } \\
\text { trabalho e participam ern açöes } \\
\text { conjuntas nas Unidades Locais de } \\
\text { Saude }\end{array}$ \\
\hline $\begin{array}{l}\text { Reconhecimento } \\
\text { profissional e social dos } \\
\text { profissionais de atenção } \\
\text { primária }\end{array}$ & $\begin{array}{l}\text { Pouco reconhecimento por parte dos } \\
\text { demais especialistas; iniciativas } \\
\text { municipais de valorização dos } \\
\text { profissionais de atenção primária }\end{array}$ & $\begin{array}{l}\text { Até à existéncia da especialidade de } \\
\text { Medicina Familiar a atenção primária } \\
\text { era muito pouco valorizada pois o } \\
\text { médico limitava-se a renovar } \\
\text { prescriçōes. No entanto agora o } \\
\text { médico da ateação primária é } \\
\text { também um especialista que } \\
\text { acoempanha o paciente e toda a sua } \\
\text { familia e que se interessa pelo setu } \\
\text { estado de saúde. Isto é agora } \\
\text { reconhecido sobretudo nas Unidades } \\
\text { Locais de Saúde }\end{array}$ \\
\hline $\begin{array}{l}\text { Iniciativas que favoreçam } \\
\text { conhocimento pessoal e } \\
\text { intercimbio de experièncias } \\
\text { entre os niveis de atençio }\end{array}$ & $\begin{array}{l}\text { Tele-consulta e tele-conferencia; } \\
\text { territorialização dos serviços da } \\
\text { ateação especializada; } \\
\text { "matriciamento" com interconsulta, } \\
\text { sessōes clinicas e capacitaçŏes }\end{array}$ & $\begin{array}{c}\text { Reuniōes de equipe; integração de } \\
\text { médicos da atenção primária na } \\
\text { urgència bospitalas; partilha de } \\
\text { enfermeiros e outros técnicos; sessōes } \\
\text { clinicas e outras reuniōes de } \\
\text { formaçăo }\end{array}$ \\
\hline
\end{tabular}

" Resultados obtidos por Almeida et al. (4)

Fuente: elaboração própria.

\section{e) Principais dificuldades para implantação de estratégias pró-coordenação}

Colocando uma questão mais aberta foram obtidos alguns testemunhos sobre as dificuldades para a implantação de estratégias pró-coordenação foram obtidas diferentes.

No Brasil, segundo Almeida et al. (11) as principais dificuldades passam por: a) insuficiente integração entre prestadores municipais e estaduais; b) baixa qualidade dos processos de regulação; c) insuficiente oferta da atenção especializada; d) ausência de políticas para a média complexidade por parte do Ministério da Saúde; e) pouco conhecimento dos processos de trabalho em atenção primária à saúde e de reconhecimento dos profissionais; f) rotatividade de profissionais.

Em Portugal, as principais dificuldades passam por: a) falta de incentivos partilhados entre ambos os níveis; aumento do trabalho burocrático realizado por médicos (registros, 
encaminhamento de pedidos, renovação de medicação); falta de reforço na atenção especializada para dar resposta em tempo adequado a todas as solicitações da atenção primária evitando recorrer ao sector privado convencionado (processo de internalização que ainda não está concluído).

\section{Discussão}

A integração entre a atenção primária e especializada é uma forte aposta e um desafio para ambos os países que já deu os primeiros passos mas que precisa de ser consolidada.

Em Portugal, verifica-se um processo com estratégias de integração mais completas ao nível da relação entre os vários profissionais, particularmente desde o reconhecimento da especialidade de Medicina Geral e Familiar (equivalente à especialidade de Medicina de Família e comunidade). Foi consensual entre os entrevistados portugueses que a integração foi desde cedo acompanhada pelo desenvolvimento de sistemas de informação que facilitaram o encaminhamento dos pacientes e a marcação de consultas. Estes resultados vão ao encontro dos achados de Monteiro (19) que analisou a adoção de tecnologias de informação e comunicação em Portugal que se iniciaram ainda nos anos 90 e concluiu que eram facilitadoras dos processos de integração e partilha de informação entre profissionais de saúde de diferentes níveis. Contudo, segundo Almeida et al. (4) no Brasil, a história clínica informatizada é ainda incipiente.

Para os resultados positivos alcançados em Portugal, contribuiu a territorialização dos serviços de atenção especializada e da atenção primária que localizando-se em área de proximidade e sobre uma mesma equipe de gestão (caso das Unidades Locais de Saúde) permitem uma maior cooperação entre as equipas. Essa relação possibilita uma maior ligação entre ambas as assistências, que segundo os achados de Santana e Costa (1) representa uma forma perfeita de integração vertical.

Nas regiões estudadas, embora tenham sido desenvolvidos protocolos com circuitos para referência dos pacientes ou para o hospital local foi esquecido o reforço da capacidade de resposta desses mesmos hospitais (mais equipamentos, mais médicos, mais salas operatórias) e houve necessidade de contratar o setor privado quando não eram cumpridos os tempos máximos de resposta garantida (termo usado em Portugal para indicar os tempos máximos de espera dos pacientes por um exame, consulta ou cirurgia), ou seja gerou-se desigualdades e retardou-se o atendimento, embora os doentes considerados muito prioritários tenham acesso garantido como é o caso de diagnóstico de câncer. Esta situação é retratada por Simões et al. (2) que faz uma análise ao desenvolvimento do sistema de saúde português e critica o acesso. Nos municípios brasileiros estudados por Almeida et al. (11), é promovida a inclusão dos casos prioritários, embora também como em Portugal existam problemas de oferta do lado da atenção especializada problemas de oferta insuficiente de atenção especializada. 
Por outro lado os processos de referência e encaminhamento são em ambos os países seguidos por um conjunto de protocolos clínicos acordados entre os diferentes níveis que permitem uma melhor referência dos pacientes. É de reconhecimento internacional e vem descrito na literatura (20) que o estabelecimento de protocolos clínicos é uma boa prática. Contudo, ainda assim, segundo Grimshaw et al. (21) por vezes as referências da atenção primária para a especializada podem ser realizadas por excesso de zelo do médico e por isso defendem que a busca de uma segunda opinião e nos seus achados concluem que a discussão prévia de casos clínicos pode reduzir as taxas de referências e permitir assim uma melhor resposta para as referências que são mesmo necessárias.

Como resultado do longo desenvolvimento das tecnologias de informação, os processos de referência em Portugal (da atenção primária para a especializada ou da especializada para a primária) tem elevados graus de qualidade e são obrigatórios. Contudo no Brasil, segundo Almeida et al. (4) a maioria dos processos de contrareferência tem diferentes graus de qualidade e regularidade o que representa um problema persistente que reflete problemas de comunicação entre os níveis de atenção.

Os entrevistados deram as suas respostas focando a importância da atenção primária com base na Declaração de Alma Ata, destacando o papel desta assistência no terreno, nomeadamente como porta de entrada para o sistema, como centro de atendimento por uma equipa de saúde, como base para a realização de exames e outros meios complementares, como forma de proximidade. Faltou a referência a outros aspetos que de acordo com a Declaração de Astana, são fundamentais para uma discussão mais ampla e para o desenvolvimento da rede de atenção primária, e que envolvem os tomadores de decisão (políticos) e a comunidade.

\section{Considerações finais}

Com esta pesquisa pretendeu-se comparar os processos e estratégias de integração entre a atenção primária à saúde e a atenção especializada, partindo dos recentes resultados relativos ao Brasil achados em um estudo realizado por Almeida et al. (4) e comparando-os com o caso português realizado com a mesma metodologia aplicada em três regiões de Portugal (Norte, Centro e Alentejo) que têm dado grandes passos na integração com a criação de um modelo inovador denominado por Unidade Local de Saúde.

De acordo com os resultados obtidos se concluiu que as experiencias desenvolvidos em ambos os países embora tenham diferentes graus de desenvolvimento apresentam pontos comuns como são exemplo a territorialização dos serviços de saúde, o entendimento que a atenção primária deve ser a principal porta de entrada, o papel de filtro do médico de família, a referenciação dos pacientes baseada em protocolos clínicos, o reconhecimento da medicina da família como especialidade médica de medicina da família. Porém, nos municípios brasileiros investigados por Almeida et al. (4) destaca-se os sistemas descentralizados de regulação que realizam o monitoramento e acompanhamento do percurso dos pacientes e das listas de espera. Do lado português, destaca-se o elevado grau de desenvolvimento dos processos clínicos eletrónicos 
que acompanharam o desenvolvimento da integração, existindo por isso aplicações que fazem a referenciação e integram o paciente numa lista de espera por prioridades e ainda em Portugal destaca-se o fato dos profissionais terem contacto próximo e serem geridos por uma mesma equipe de gestão que promove a internalização dos exames de diagnóstico e das consultas.

Em conclusão e ao encontro da Declaração de Astana, conclui-se que tanto no Brasil como em Portugal é necessário direcionar as opções políticas em saúde para a atenção primária, integrar a saúde com os restantes sectores de atividade, construir uma rede de atenção primária sustentável e articulada com os restantes níveis de resposta, capacitar cidadãos e as comunidades para a importância da atenção primária e alinhar o interesse dos cidadãos e a satisfação das suas necessidades com as políticas, estratégias e planos de saúde.

\section{Referências}

1. Santana R, Costa C. A integração vertical de cuidados de saúde: aspectos conceptuais e organizacionais. Rev. Port. Saúde Pública. 2008;7(1):29-56.

2. Simões J, Augusto G, Fronteira I. Introduction of freedom of choice for hospital outpatient care in Portugal: Implications and results of the 2016 reform. Health Policy. 2017;121(12):1203-1207.

3. Organização para a Cooperação e Desenvolvimento Económico. Health Status [Internet]. 2018 [revisão 2018 jun. 16]. Disponível em: http://stats.oecd.org/

4. Almeida $\mathrm{P}$, Gérvas J, Freiere J, Giovanella, L. Estratégias de integração entre atenção primária à saúde e atenção especializada: paralelos entre Brasil e Espanha. Saúde Debate. 2013;37(98):400-415.

5. Brasil. Ministério da Saúde, Secretaria de Atenção à Saúde, Departamento de Atenção Básica. Política nacional de atenção básica. Brasília: Editora Ministério da Saúde; 2006.

6. Nunes A. Direito à saúde em Portugal: delimitação jurídica do SNS. Direitos Fundam. Justiça. 2017;11(37):17-34.

7. Biscaia A, Heleno L. A Reforma dos Cuidados de Saúde Primários em Portugal: portuguesa, moderna e inovadora. Ciênc. Saúde Colet. 2017;22(3):701-711.

8. Nunes A, Matos A. Experiências de Gestão Hospitalar Realizadas em Portugal: Uma Perspectiva Comparada. Rev. Eletrônica Gest. Saúde. 2017;8(2):284-301.

9. Portugal. Diário da República. Decreto-Lei n. ${ }^{\circ}$ 207/99, Cria a Unidade Local de Saúde de Matosinhos n. ${ }^{\circ}$ 133/Série I-A; 1999 jun. 9.

10. Simões J. Retrato político da saúde. Dependência do percurso e inovação em saúde: da ideologia ao desempenho. Coimbra: Almedina; 2004.

11. Almeida $\mathrm{P}$, Giovanella L, Mendonça $\mathrm{M}$, Escoriel S. Desafios à coordenação dos cuidados em saúde: estratégias de integração entre níveis assistenciais em grandes centros urbanos. Cad. Saúde Pública. 2010;26(2):286-298. 
Integração entre atenção primária à saúde e atenção especializada: paralelos entre a experiencia Brasileira e Portuguesa

12. Organização Pan-Americana de Saúde. Integrated health service delivery networks: concepts, policy options and a road map for implementation in the Americas [Internet]. Washington (DC): OPAS Publications; 2011 [revisão 2018 maio 24]. Disponível em: http://new.paho.org/hq/dmdocuments/2 011/PHC_IHSD-2011Serie4.pdf

13. Sobczak A. Opportunities for and constraints to integration of health services in Poland. Int. J Integr. Care. 2010;2(1):1-10.

14. Byrne M, Ashton C. Incentives for vertical integration in healthcare: the effect of reimbursement systems. J Healthc. Manag. 1999;44(1):34-46.

15. Leichsenring K. Developing integrated health and social care services for older persons in Europe. Int J Integr Care. 2004;4(3):1-15.

16. Tsutsui T. Implementation process and challenges for the community-based integrated care system in Japan. Int J Integr Care. 2014;14:e02.

17. Ahgren B, Axelsson R. A decade of integration and collaboration: the development of integrated health care in Sweden 2000-2010. Int J Integr Care. 2011;11:e007.

18. Portugal. Serviço Nacional de Saúde. Tempos Médios De Espera [Internet]. Disponível em: http://tem pos.min-saude.pt/\#/instituicoes

19. Monteiro M. A Adopção da eHealth nos Hospitais Públicos em Portugal, 1996-2007. Lisboa: Instituto Superior de Ciências Sociais e Políticas; 2011.

20. Martínez D, Navarrete M, Lorenzo I. Factores que influyen en la coordinación entre niveles asistenciales según la opinión de directivos y profesionales sanitarios. Gac Sanit. 2009;23(4):280-286.

21. Grimshaw J, Winkens R, Shirran L, Cunningham C, Mayhew A, Thomas R, et al. Interventions to improve outpatient referrals from primary care to secondary care. The Cochrane Database Syst Rev. 2005; 20(3): CD005471.

Notas

* $\quad$ Artigo de revisão 